Article

\title{
Cation/Proton Antiporter Genes in Tomato: Genomic Characterization, Expression Profiling, and Co-Localization with Salt Stress-Related QTLs
}

\author{
Zahid Hussain ${ }^{1, *}$, Habiba Khan ${ }^{2}$, Muhammad Imran ${ }^{3} \mathbb{D}$, Muhmmad Kashif Naeem ${ }^{4}$, Sabir Hussain Shah ${ }^{5}$, \\ Arshad Iqbal ${ }^{1}$, Syed Shujait Ali ${ }^{1}$, Muhammad Rizwan ${ }^{1}$, Shahid Ali ${ }^{1}$, Muhammad Atif Muneer ${ }^{6}$, \\ Emilie Widemann ${ }^{7}$ (D) and Sarfraz Shafiq ${ }^{8, *}$
}

check for updates

Citation: Hussain, Z.; Khan, H.; Imran, M.; Naeem, M.K.; Shah, S.H.; Iqbal, A.; Ali, S.S.; Rizwan, M.; Ali, S.; Muneer, M.A.; et al. Cation/Proton Antiporter Genes in Tomato: Genomic Characterization, Expression Profiling, and Co-Localization with Salt Stress-Related QTLs. Agronomy 2022, 12, 245. https://doi.org/10.3390/ agronomy12020245

Academic Editor: Juan

J. Gutierrez-Gonzalez

Received: 17 December 2021

Accepted: 15 January 2022

Published: 19 January 2022

Publisher's Note: MDPI stays neutral with regard to jurisdictional claims in published maps and institutional affiliations.

Copyright: (C) 2022 by the authors. Licensee MDPI, Basel, Switzerland. This article is an open access article distributed under the terms and conditions of the Creative Commons Attribution (CC BY) license (https:// creativecommons.org/licenses/by/ $4.0 /)$.
1 Center for Biotechnology and Microbiology, University of Swat, Mingora 19200, Pakistan; arshad.iqbal@uswat.edu.pk (A.I.); shujaitswati@uswat.edu.pk (S.S.A.); muhammad.rizwan@uswat.edu.pk (M.R.); shahidali@uswat.edu.pk (S.A.)

2 Department of Biotechnology, Quaid-i-Azam University, Islamabad 45320, Pakistan; habibazahidd@gmail.com

3 State Key Laboratory for Conservation and Utilization of Subtropical Agro-Bioresources, College of Agriculture, South China Agriculture University, Guangzhou 510642, China; imran_m1303@yahoo.com

4 National Institute for Genomics and Advanced Biotechnology (NIGAB), National Agricultural Research Center (NARC), Park Road, Islamabad 45320, Pakistan; kashifuaar@gmail.com

5 Department of Agricultural Sciences, Faculty of Sciences, Allama Iqbal Open University, Islamabad 45320, Pakistan; sabir.hussain@aiou.edu.pk

6 College of Resources and Environment/International Magnesium Institute, Fujian Agriculture and Forestry University, Fuzhou 350002, China; M_atifmuneer@yahoo.com

7 Institut de Biologie Moléculaire des Plantes, CNRS-Université de Strasbourg, 67084 Strasbourg, France; Ewidema4@uwo.ca

8 Department of Anatomy and Cell Biology, University of Western Ontario, 1151 Richmond St., London, ON N6A 5B8, Canada

* Correspondence: zahid@uswat.edu.pk (Z.H.); sshafiq2@uwo.ca (S.S.)

\begin{abstract}
The cation/proton antiporter (CPA) family represents a class of transmembrane transporter proteins that play a crucial role in plants during high salinity stress by maintaining the cell's ionic balance and $\mathrm{pH}$ homeostasis. So far, the CPA genes have not been systematically characterized in tomato (Solanum lycopersicum). In this study, we identified and analyzed 33 putative CPA genes in tomato. Phylogenetic analysis showed that tomato CPAs could be classified into three subgroups, i.e., CHX (18 genes), KEA (8 genes), and NHX (7 genes). CPA genes within each subgroup shared similar motifs, conserved catalytic domains and gene structure. Further analysis revealed that the CPA genes were unevenly distributed on the chromosomes and segmental duplication events played a major role in the expansion of the CPA gene family in tomato. Gene expression analysis exhibited that CPA genes were differentially expressed in different tissues, various stages of fruit development, and differentially regulated in response to abiotic stresses, especially salt stress. Further, co-localization of tomato CPA genes with quantitative trait loci (QTL) of salt stress-related phenotypes revealed their broader functions in salt stress tolerance. Finally, predicted protein-protein interactions of tomato CPAs, gene ontology analysis, and the presence of putative cis-elements in the promoter further support the diverse role of tomato CPAs in plant development and plant stress tolerance. In brief, this study highlights the potential role of tomato CPAs in plant development and abiotic stress tolerance, especially in salt stress, and provides comprehensive information to explore new candidate genes for salt tolerance in tomato.
\end{abstract}

Keywords: CPA; CHX; KEA; NHX; ion transporter; tomato; stress response; stress QTLs; expression analysis 


\section{Introduction}

The cation/proton antiporters (CPAs) are responsible for maintaining the $\mathrm{pH}$ homeostasis and ion concentrations in all living species, including plants and animals. CPA genes play critical roles in many aspects of plant growth, development, signal transduction, and stress responses [1]. The CPA members are structurally divided into two broad categories, designated as CPA1 and CPA2 [2]. The CPA1 includes the $\mathrm{Na}^{+} / \mathrm{H}^{+}$exchanger (NHX) family, while CPA2 consists of $\mathrm{K}^{+}$efflux antiporter (KEA) and cation $/ \mathrm{H}^{+}$exchanger (CHX) family of proteins $[3,4]$.

The CPA superfamily is functionally involved in the exchange and transport of monovalent cations in plants [5], therefore, can be divided into two main types, i.e., transport of sodium ion $(\mathrm{Na}+)$ or potassium ion $\left(\mathrm{K}^{+}\right)$and a cation exchange for one or two protons [6]. In plants, the salt tolerance mechanism is mainly based on maintaining the ion homeostasis, osmotic balance, and cellular tolerance. Plants respond to the elevated level of ions $\left(\mathrm{Na}^{+}\right.$, $\mathrm{Cl}^{-}$, and $\mathrm{K}^{+}$) by regulating the transport of water and ions between the plant and the environment, as well as distribution inside the plant, and among the organelles [7]. In plants, ion concentration and $\mathrm{pH}$ balance feat are accomplished with a diverse array of transporters, especially the CPA proteins [1] that are localized in the plasma membrane as well as organelle membranes such as endosomes, vacuoles, and chloroplasts $[3,7,8]$. The CPA gene family has been extensively studied in various plant species, including Arabidopsis thaliana [9], radish (Raphanus satious) [10], wheat (Triticum aestivum) [5], rice (Oryza sativa) [11], grape (Vitis vinifera) [12], and pear (Pyrus bretschneideri) [13]. However, the function of CPAs in tomato remains largely unknown.

Tomato (Solanum lycopersicum) is considered the most important vegetable crop cultivated worldwide. Tomato has a relatively small and compact genome of approximately $950 \mathrm{Mb}$ and a short life cycle [14]. Tomato is a rich source of nutrients and a model plant for fleshy fruit development. In the last decade, the genome of tomato and its wild relatives have been completely sequenced by the international genome sequencing consortium [15]. However, with a continuous expansion in the cultivation of tomato, its production has been seriously impaired in recent years by various abiotic stresses, such as temperature, drought, and salinity [16]. The tomato plant is highly vulnerable to salinity stress because high levels of $\mathrm{Na}+$ ions adversely affects cellular metabolism and ion homeostasis. Therefore, the identification of potential genes that could confer resistance to abiotic stresses is of the utmost importance for molecular breeding of tomato and a comprehensive understanding of CPA gene family functions in tomato would be an ideal beginning based on the reported role of CPA genes in other plant species.

In the present study, a comprehensive genome-wide analysis of the Solanum lycopersicum CPAs, hereafter referred to as SlCPAs, gene family was performed and identified 33 SICPA genes. Moreover, they were further analyzed to determine their phylogenetic relationship, physiochemical parameters, conserved motifs, subcellular localization, and gene structure. The transcriptome of SICPA genes was evaluated in different tissues, various fruit developmental stages, and under abiotic stresses. Furthermore, we carried out the co-localization of SICPA genes with QTLs of salt stress-related phenotypes. Finally, protein-protein interactions among the SlCPAs and the associated proteins were predicted. This study provides a fundamental understanding of SlCPAs in conferring abiotic stress resistance in tomato and will be useful for the long-term improvement of stress tolerance in tomato.

\section{Materials and Methods}

\subsection{Identification of CPA Gene Family in Tomato}

Genomic and amino acid sequences of tomato CPA family members were retrieved from the Phytozome database (https: / / phytozome.jgi.doe.gov/pz/portal.html, accessed on 25 march 2021). The Arabidopsis CPA protein sequences were obtained from TAIR10 database (http:/ / www.arabidopsis.org, accessed on 25 March 2021) [17]. HMM profile (Hidden Markov Model) was built from Arabidopsis CPA proteins to identify tomato CPAs 
using HMMER 3.0. Then, the HMM profile was used to query the tomato protein database (E-value less than $\left.\mathrm{e}^{-10}\right)[18,19]$. The putatively identified SICPA sequences were verified by BLASTp search at e-value $<1.0$. The SlCPA protein sequences were verified by a conserved domain search (PF00999) using InterPro webtool (http:/ / www.ebi.ac.uk/x/pfa/iprscan/, accessed on 30 March 2021) [20]. We eliminated the proteins without the CPA-conserved domain. The CDA-Hit-v4.6.648 (cluster database at high identity with tolerance) with a threshold identity of $90 \%$ further removed the redundant proteins sequences and provided $33 \mathrm{SlCPA}$ representative proteins for further study.

\subsection{Physiochemical Properties and Sequence Analysis}

The physiochemical details of SlCPA proteins were predicted using the ExPASy ProtParam tool (http:/ / web.expasy.org/protparam/, accessed on 10 April 2021).) [21]. The various properties include isoelectric point, length of protein, protein molecular weights, theoretical pI, GRAVY, and instability index. The subcellular localization of SlCPA proteins were detected using CELLO2GO (http:/ / cello.life.nctu.edu.tw/cello2go/, accessed on 10 April 2021) [22]. Additionally, transmembrane helices in SlCPA proteins were analyzed by TMHMM Server v.2.0 (http:/ / www.cbs.dtu.dk/services/TMHMM/, accessed on 12 April 2021) [23]. The gene structures of SlCPAs (exon/intron) were displayed by the GSDS server (http:/ /gsds.gao-lab.org, accessed on 15 April 2021). The conserved motifs of SlCPA proteins were analyzed using the MEME server with default parameters (http:/ / meme-suite.org/tools/meme, accessed on 15 April 2021) [24].

\subsection{Phylogenetic Analysis}

All the CPA protein sequences from tomato and Arabidopsis were aligned with Clustal $X$. Phylogenetic tree was built by the neighbor-joining (NJ) method and bootstrap consensus tree values were set to 1000 replicates. The phylogenetic analysis was executed in MEGA 7.0 [25], and the tree visualization was done by Itol v 6 (https://itol.embl.de, accessed on 25 April 2021) [26].

\subsection{Chromosomal Localization and Synteny Analysis}

The genome and annotation files of tomato and Arabidopsis were downloaded from the phytozome website. Gene duplication events, paralogous, and orthologous genes were identified by MCScanX toolkit [27]. The collinear block was identified by SlCPAs duplication events in the MCScanX. The synteny blocks were constructed by utilizing TBtools [28]. The chromosomal positions of SICPA genes were identified according to the Phytozome database and the chromosomal map was visualized using the Ritchielab phenogram tool (http:/ / visualization.ritchielab.org/phenograms/plot, accessed on 2 May 2021).

\subsection{Promoter Analysis}

The presence of cis-regulatory elements in the SICPA genes was investigated as described previously [29]. Briefly, promoter sequences (2 kb upstream genomic DNA sequences) of SlCPA genes were analyzed using the PlantCARE database [30] and validated in the PLACE database [31] and visualized into TBtools [28].

\subsection{Transcriptomic Data Analysis of SICPA Genes in Different Tissues and under Different Abiotic Stresses}

Illumina high throughput RNA-sequencing data of leaves, roots, flower buds, fully opened flowers, and $1 \mathrm{~cm}, 2 \mathrm{~cm}$, and $3 \mathrm{~cm}$ of mature green, breaker, and breaker +10 fruits of tomato cultivar Heinz were downloaded from the Tomato Functional Genomics Database (http:/ / ted.bti.cornell.edu/cgi-bin/TFGD/digital/home.cgi, accessed on 10 May 2021). Illumina high throughput RNA-sequencing data of tomato under heat and drought (GEO accession: GSE151277), and salinity (GEO accession: GSE148353) were downloaded from the NCBI GEO database. The expression data in three replicates were used for the transcrip- 
tional profiling of SICPA genes in different tissues, developmental stages, and under abiotic stresses. The FPKM (fragments-per-kilobase-per-million) protocol was utilized for the expression analysis of each SICPA gene. The heatmap charts were drawn by the pheatmap package in R Studio.

\subsection{Plant Material and Salinity Stress Treatment}

The tomato cultivar (Solanum lycopersicum L. cv. Rio Grande) was used to analyze the expression patterns of SICPA genes in response to salinity stress. Seeds were planted in the growth room at $25 / 22{ }^{\circ} \mathrm{C}$ (day/night), 60\% humidity, 12,000 lx light, and 16/8 h light and dark regime. After germination, seedlings of uniform length were shifted to $1 / 5$ Hoagland solution with pH 5.0 as previously described [32], under the same growth conditions. After six days, the nutrient solution was supplemented with $250 \mathrm{mM} \mathrm{NaCl}$ for salinity treatment.

\subsection{RNA Isolation, $c D N A$ Synthesis, and qRT-PCR}

For RNA isolation, leaf tissues were collected after 0, 3, 6, 12, 24, 48 and 96 h of salinity stress. RNA from leaves was isolated using RNA Plant Mini Kit (Tiangen, Beijing, China). The RNA was converted to cDNA using the PrimeScriptTM RT kit (Takara, Kusatsu shi, Japan). qRT-PCR analysis was performed using LightCycler ${ }^{\circledR} 480$ System (Roche, Basel, Switzerland) using gene-specific primers (Supplementary Materials, Table S3) as described previously in [33]. The relative expression level was calculated by the $2^{-\Delta \Delta C T}$ formula and the data were normalized with Actin (Act). Three biological replicates were performed for each sample.

\subsection{Gene Ontology and Network Interaction Analysis}

Gene ontology (GO) terms of SlCPA genes were determined using CELLO2GO web server for protein subcellular localization prediction with functional gene ontology annotation (http: / / cello.life.nctu.edu.tw / cello2go/, accessed on 25 May 2021). The proteinprotein interactions of stress-responsive SlCPAs were carried out using STRING 9 (https: / / string-db.org, accessed on 25 May 2021) [34].

\subsection{Co-Localization of SICPAs with Salt Stress-Related QTLs}

To identify the localization of QTLs for morphological and biochemical traits under salt stress, QTLs and linked molecular markers were retrieved from the Sol Genomics Network website (https:/ / solgenomics.net/search/phenotypes/qtl, accessed on 1 June 2021) and tomato marker database (http:/ / marker.kazusa.or.jp/Tomato/, accessed on 1 June 2021) as well as from the publications [16,35-38]. The markers of respective QTLs were obtained from previous publications and co-localization was shown as described previously [39]. Briefly, each marker sequence or name was BLAST against Sol Genomics Network website (https: / / solgenomics.net/search/phenotypes/qtl, accessed on 1 June 2021) and tomato marker database (http:/ / marker.kazusa.or.jp/Tomato/, accessed on 1 June 2021) to obtain the physical position. SICPA genes co-localized with salt stress-related QTLs were displayed using the MapChart software [40]. This displayed the SICPA genes distribution along with surrounding QTLs. QTLs co-localized with the genes are indicated by asterisk and purple color.

\section{Results}

\subsection{Identification and Characterization of CPAs in Tomato}

To identify the CPAs in tomato, Hidden Markov Model (HMM) search was carried out against the tomato genome. The redundant protein sequences were removed by the CD-Hit program, and 33 non-redundant CPA genes were obtained. These tomato CPA genes were named SlCHX1-18, SlNHX1-7, and SlKEA1-8 according to their distributions and relative linear orders among their respective chromosomes. Basic information of all SlCPA proteins (gene name, chromosome, protein length, molecular weight, theoretical pI, aliphatic index, GRAVY, subcellular localization, and number of predicted transmembrane 
helices) is provided (Supplementary Materials Table S1). Notably, all these SlCPA proteins contained a Na${ }^{+} / \mathrm{H}^{+}$exchanger domain, with the protein length of 229 aa to 1199 aa and the molecular weight ranging from $24 \mathrm{kDa}$ to $128 \mathrm{kDa}$. The SlCPA proteins contain 7-14 transmembrane domains with membranous subcellular localization.

\subsection{Phylogenetic, Conserved Motifs, and Gene Structure Analysis of Tomato CPA Genes}

The phylogenetic tree was constructed to explore the evolutionary relationship of full-length CPA protein sequences of tomato and Arabidopsis. Based on their evolutionary relationship, similar to Arabidopsis, the SlCPAs could be distinctly categorized into three main subgroups, i.e., $\mathrm{CHX}, \mathrm{KEA}$, and NHX. The CHXs were further classified into three subgroups-C1, C2, and C3. Similarly, KEAs and NHXs were further divided into two subgroups-K1 and K2, and N1 and N2, respectively (Figure 1).

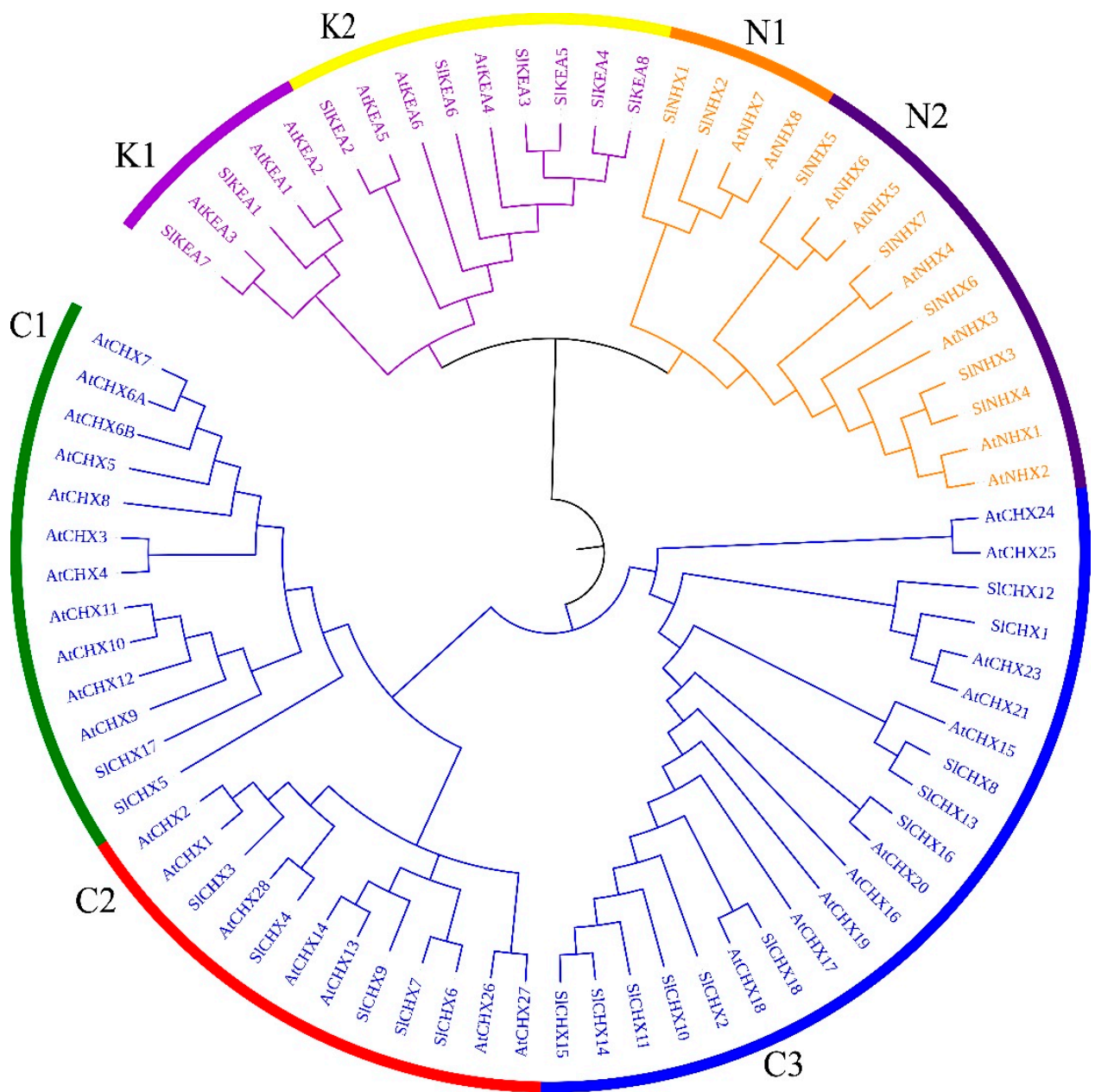

Figure 1. Phylogenetic relationship of proteins from CPA supergene family of Solanum lycopersicum and Arabidopsis. The members of each gene family are highlighted in different colors. The subgroups in each family are denoted as $\mathrm{C} 1, \mathrm{C} 2, \mathrm{C} 3$ (CHX), N1, N2 (NHX), and K1, K2 (KEA).

The $\mathrm{Na}^{+} / \mathrm{H}^{+}$exchanger domain was predicted in the $33 \mathrm{SlCPA}$ proteins by InterPro scan to verify the integrity of the proteins. Furthermore, the SlCPA protein sequences and structures were also examined by MEME to predict the conserved motif sites (0-10). Tomato $\mathrm{CHXs}$ proteins have seven to thirteen conserved motifs, while KEAs proteins have four to seven conserved motifs. In the NHX gene family, SINHX1, SINHX3, and SINHX5 contained two conserved motifs, whereas SINHX4, SINHX6, and SINHX7 have only one conserved motif (Figure 2A). We also noted that tomato NHX, KEA, and CHX subgroups share similar conserved motifs but also have different conserved motifs. For example, four motifs were 
common in both the CHX and KEA families, while one to two motifs were exclusively present in the NHX family (Figure 2A). The motif logos discovered in SICPA genes are given in Figure S1 and the motif information is provided in Table S4.
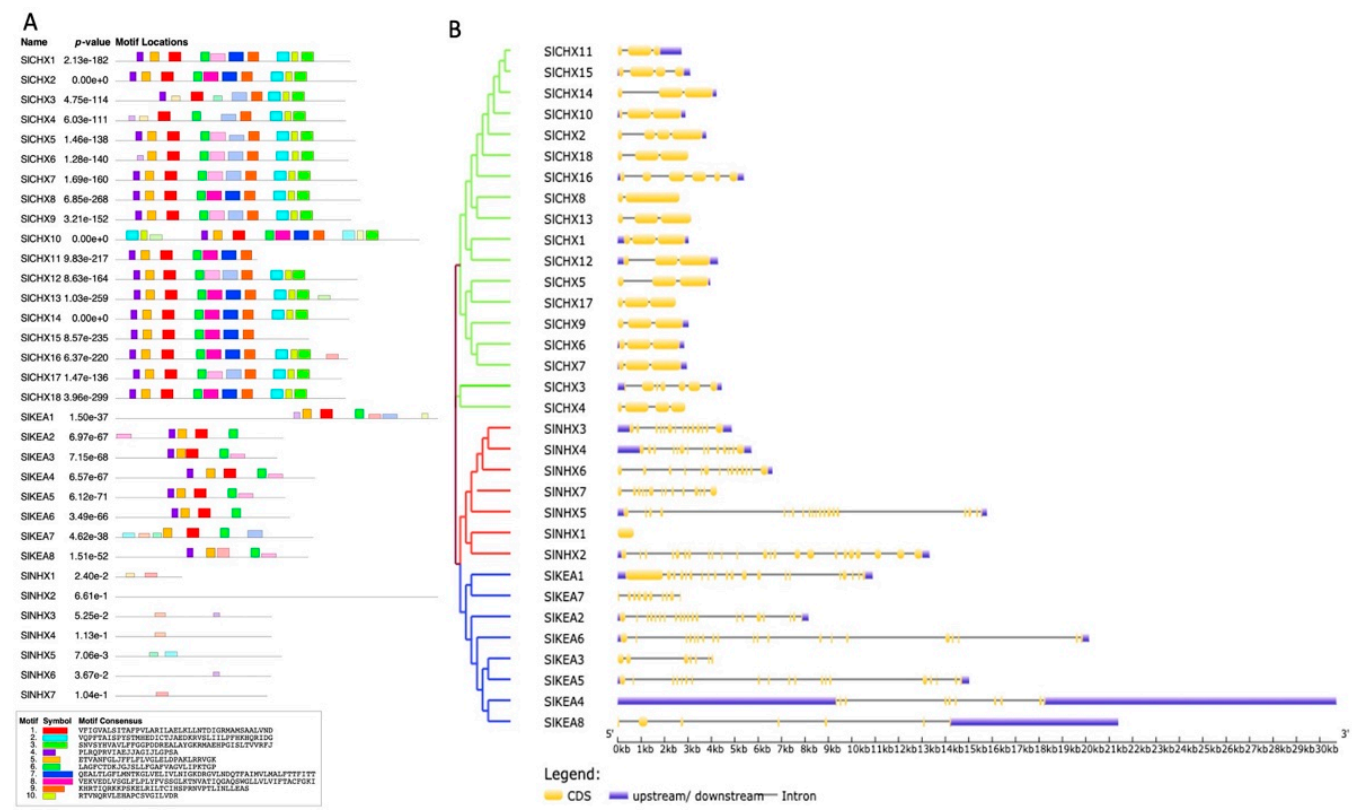

Figure 2. Conserved motif analysis and exon-intron distribution of SlCPA proteins. (A) Conserved motif distribution of SlCPA proteins. The 10 motifs are represented by different color ranges. (B) Gene structure of SICPA genes. Untranslated regions (UTRs), coding (exons), and non-coding (introns) are denoted by blue and yellow boxes, and black lines, respectively.

The gene structural diversity of SlCPAs was further investigated with exon/intron analysis (Figure $2 \mathrm{~B}$ ). The analysis shows that SICPAs vary greatly in the sequence length and the number of introns/exons. The gene structure in the same subgroup (CHX, KEA, and $\mathrm{NHX}$ ) was found similar, whereas the number and length of exons/introns were different. For example, SINHX1 has only one exon, while SINHX2 has thirteen exons. Furthermore, SlCHXs have fewer but longer exons, while SlKEAs have many but small exons. The UTRs regions were found in most SICPAs except for a few.

\subsection{Chromosomal Localization and Duplication Analysis of CPA Genes}

The locations of SICPA genes on the chromosomes were obtained from the Phytozome database and 33 SlCPA genes were successfully mapped to the 12 chromosomes of tomato (Figure 3). The SlCPA genes were found unevenly distributed on the different chromosomes of tomato, ranging from one to eight genes per chromosome. Chromosome eight possessed the highest number of genes (seven), followed by five genes on chromosome one, and four genes on chromosome six. We further investigated the contribution of gene duplication to the expansion of SICPA genes and found five segmental duplications in the tomato genome (SlNHX4/SlNHX7, SlKEA1/SlKEA7, SlKEA2/SlKEA3, SlCHX2/SlCHX11, and $\mathrm{SlCHX} 5 / \mathrm{SlCHX17).}$

Furthermore, the CPA genes of tomato and Arabidopsis were compared and analyzed by the synteny block method to explore the evolutionary mechanism of SICPA members. The syntenic map revealed many colinear genes pairs between tomato and Arabidopsis. The orthologous genes in the synteny blocks with one-to-one pairing (SIKEA6-AT5G11800, SlCHX16-AT3G53720, SIKEA2-AT5G51710, SINHX3-AT3G06370, and SINHX4-AT5G27150) revealed common ancestors of these genes in Arabidopsis and tomato. In addition, there were also gene pairs with one, two, or three Arabidopsis genes corresponding to the same or different tomato genes in the synteny blocks (SlCHX14-AT4G23700/AT5G41610/AT1G64170, SlCHX10-AT1G64170/AT4G23700/AT5G41610, SlKEA-AT1G01790/AT3G05030/AT4G00630, 
and SINHX2-AT1G14660.1). Such types of synteny events suggested that many CPA genes appeared before the divergence of the tomato and Arabidopsis lineages (Figure 4).

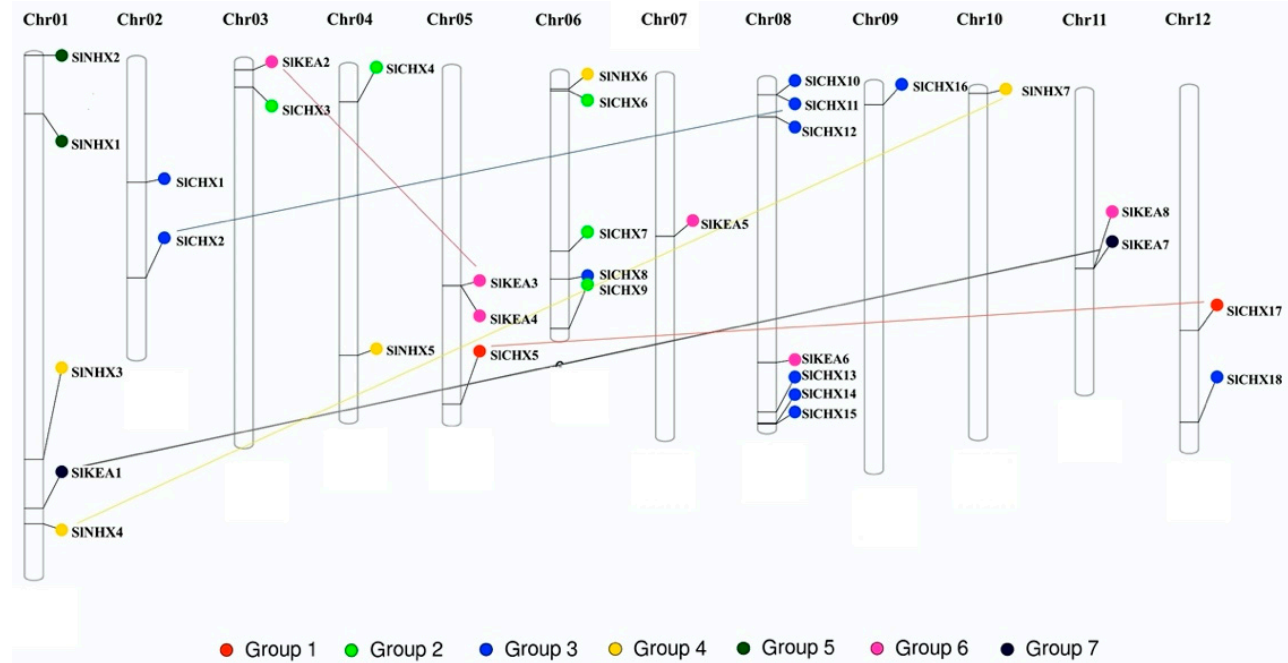

Figure 3. Distribution of tomato SlCPA genes on 12 chromosomes of tomato. The chromosome number is indicated at the top of each bar chart. Chromosomal positions of the SICPA genes are displayed by the exact name and the duplicated genes are connected with different colored lines.

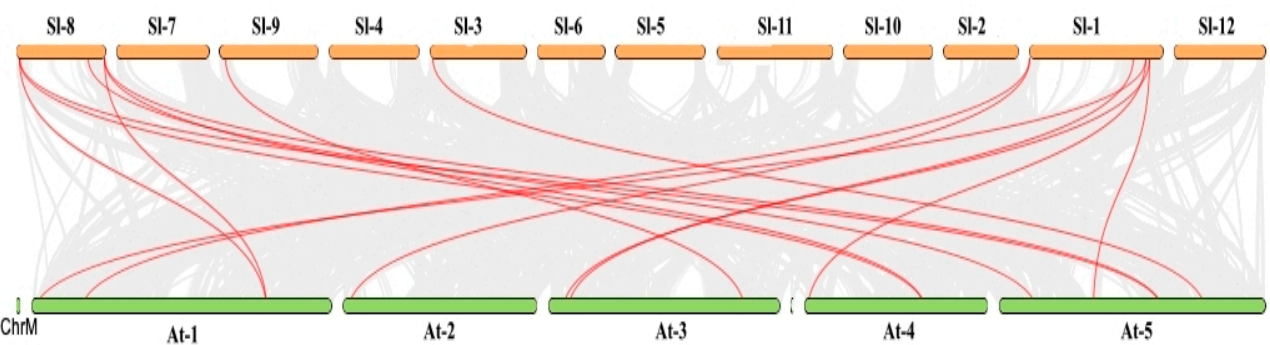

Figure 4. Evolutionary analysis of the SlCPA genes. Synteny blocks representation of CPA genes between tomato and Arabidopsis. The red lines between two chromosomal locations indicate syntenic relationship between tomato (Sl-1 to 12) and Arabidopsis (At-1 to 5).

\subsection{Putative Cis-Elements in the Promoter Regions of SlCPAs}

To gain more insights into the putative functions of SlCPAs, the presence of cisregulatory elements were scanned in the upstream $2 \mathrm{~kb}$ promoter regions of the SlCPA gene family using the Plant Care database (Figure 5, Supplementary Materials, Table S2). The result revealed that SICPAs carry TATA and CAAT box core cis-elements, light responsive, environmental stress related, development responsive, and phytohormones response elements in their promoters. This suggests that SlCPAs are potentially involved in developmental processes and environmental stress tolerance. Among the light responsive elements, I-box, ATCT-motif, and G-box motif were the most abundant cis-elements found in the SlCPAs promoters. Among the environmental stress-related cis-elements, TC-rich repeats, MBS, and LTS cis-elements were abundant in the promoter of SlCPAs. Similarly, CGTCA-motif, ABRE, and ARE were found to be the most abundant in SlCPAs promoters, while O2-site was the most abundant in development-related cis-elements. Although $C H X$, $K E A$, and NHX subgroups of SICPA genes share most of the cis-regulatory elements in their promoter regions, some cis-elements were found absent in certain groups. Notably, cis-elements were also found different in the promoter of duplicated genes, suggesting their unique functions. For example, the promoter of SIKEA2 has five light responsive ATCT-motifs, while the promoter of SIKEA3 has only one ATCT-motif cis-element. 
A
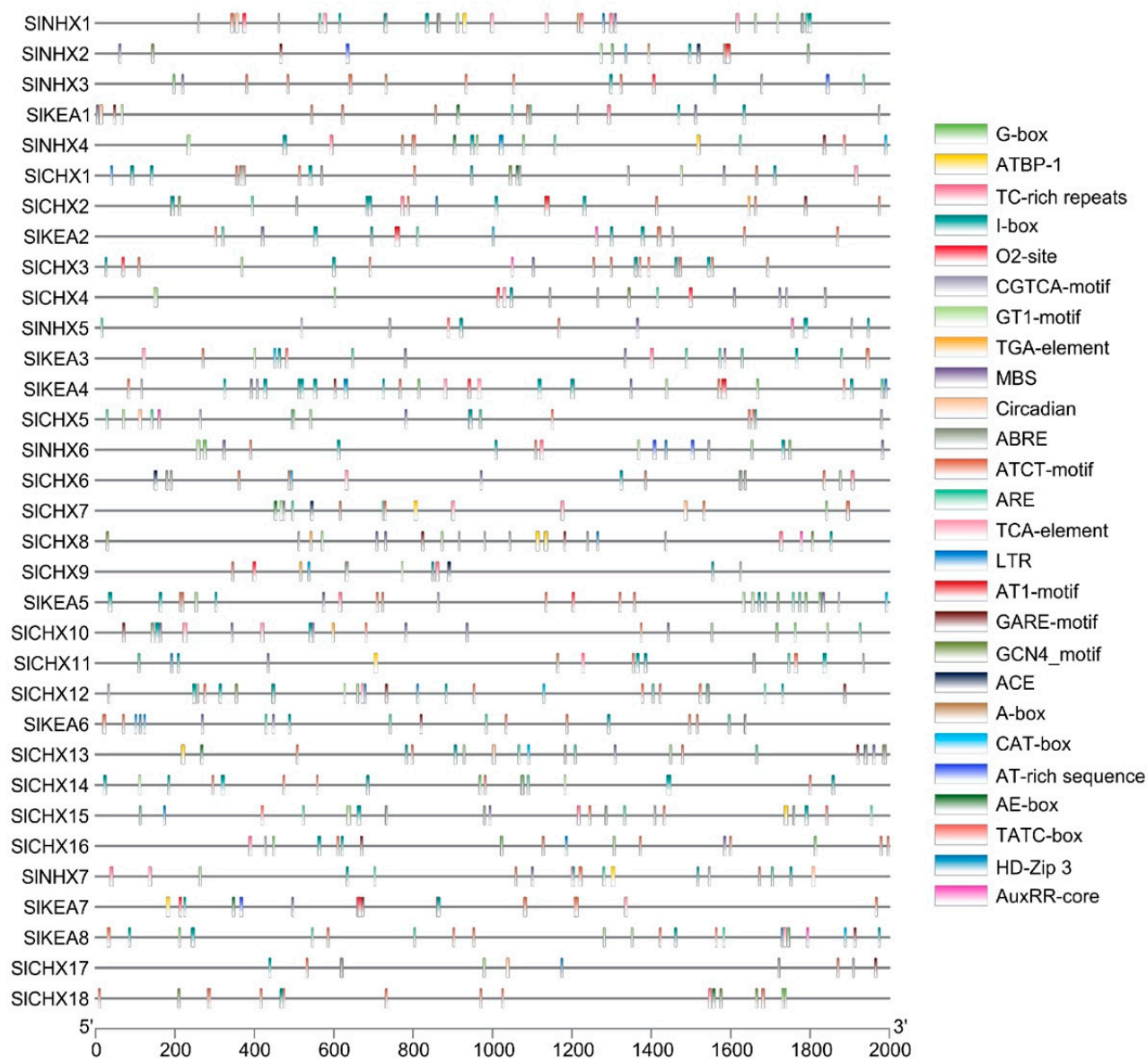

B
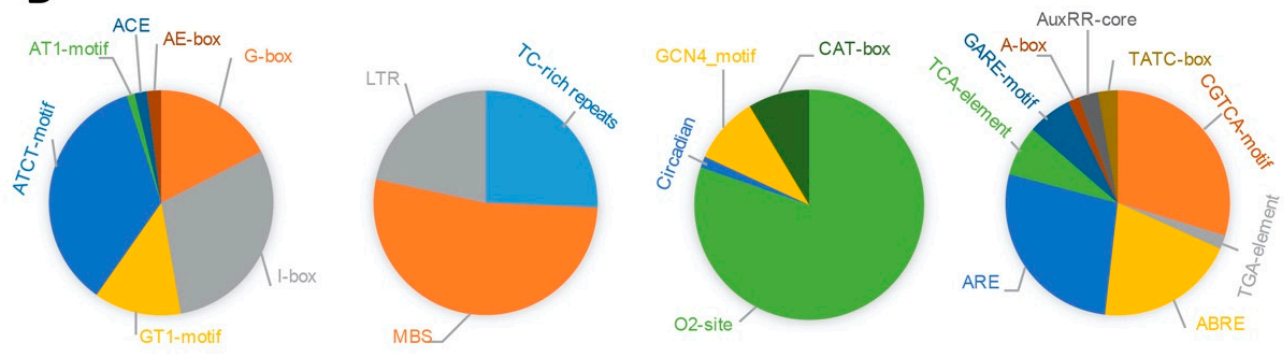

Light responsive elements Environmental stress- elements Development related elements Hormone responsive elements

Figure 5. Putative cis-regulatory elements in the promoter of SlCPAs. (A) Promoter regions $2 \mathrm{~kb}$ upstream of SICPA genes analyzed by Plant Care. Different-colored rectangles represent different ciselements. (B) Pie charts represent the classification of putative cis elements into four major categories.

\subsection{Expression Analysis of SlCPA Genes in Different Tissues and at Various Stages of Fruit Development}

To understand the potential physiological functions of SlCPAs, we investigated the expression of SICPA genes in different tissues and at various stages of fruit development by using the publicly available RNA-seq data. The results showed that SICPAs were widely expressed in leaf, root, flowers, and at various stages of fruit development (Figure 6A), suggesting the diverse biological functions of SlCPAs in different tissues and at various stages of fruit development. We also noted the tissue-specific gene expression among the different subgroups of SICPAs. For example, $C H X$ subgroup showed the highest expression in flower buds and flowers compared with roots and leaves. Similarly, KEA and NHX subgroups of SICPAs genes, such as SIKEA2, SIKEA4, SIKEA5, SlKEA6, SlNHX2, SINHX4, 
and SINHX6, showed an increase in gene expression with an increase in fruit size. These results suggest that $C H X, K E A$, and $N H X$ subgroups of SICPAs may have common as well as specific functions in tomato growth and development.

A

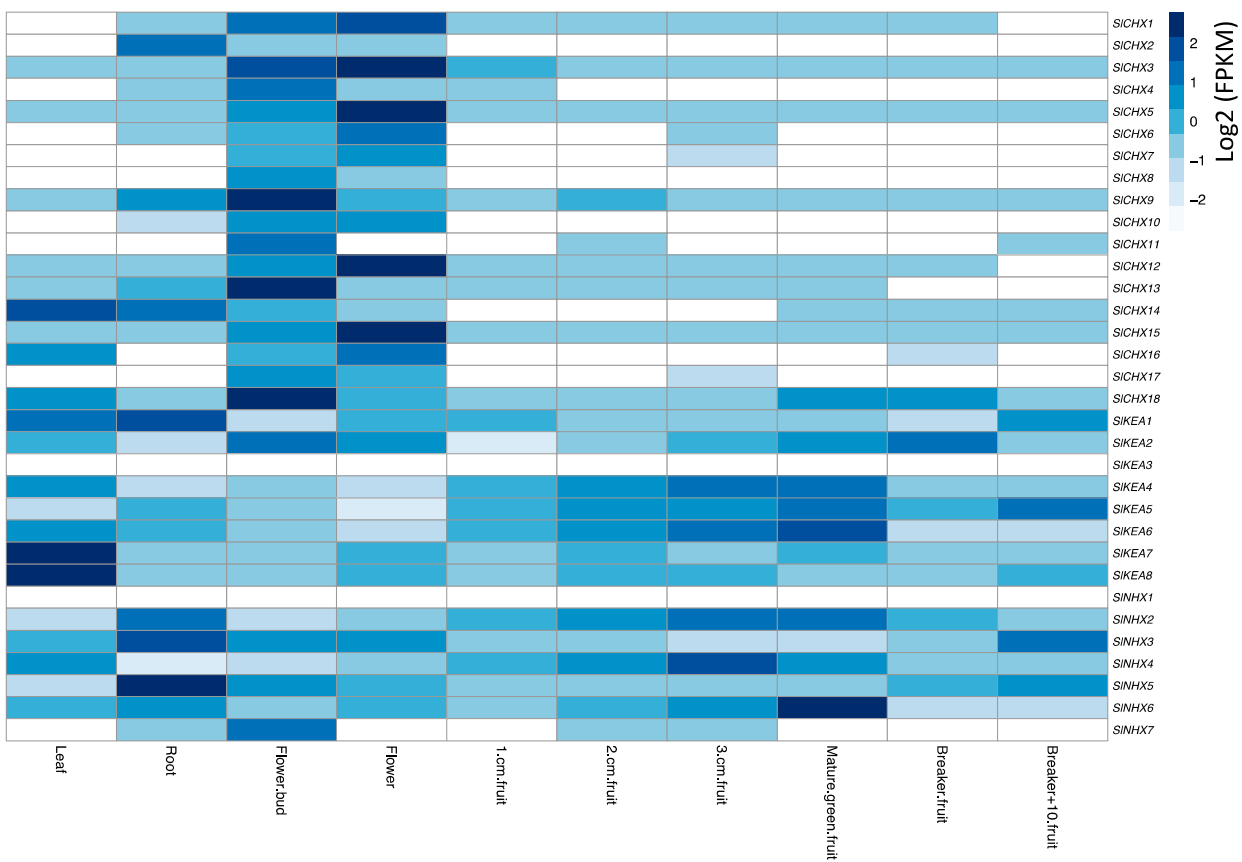

B

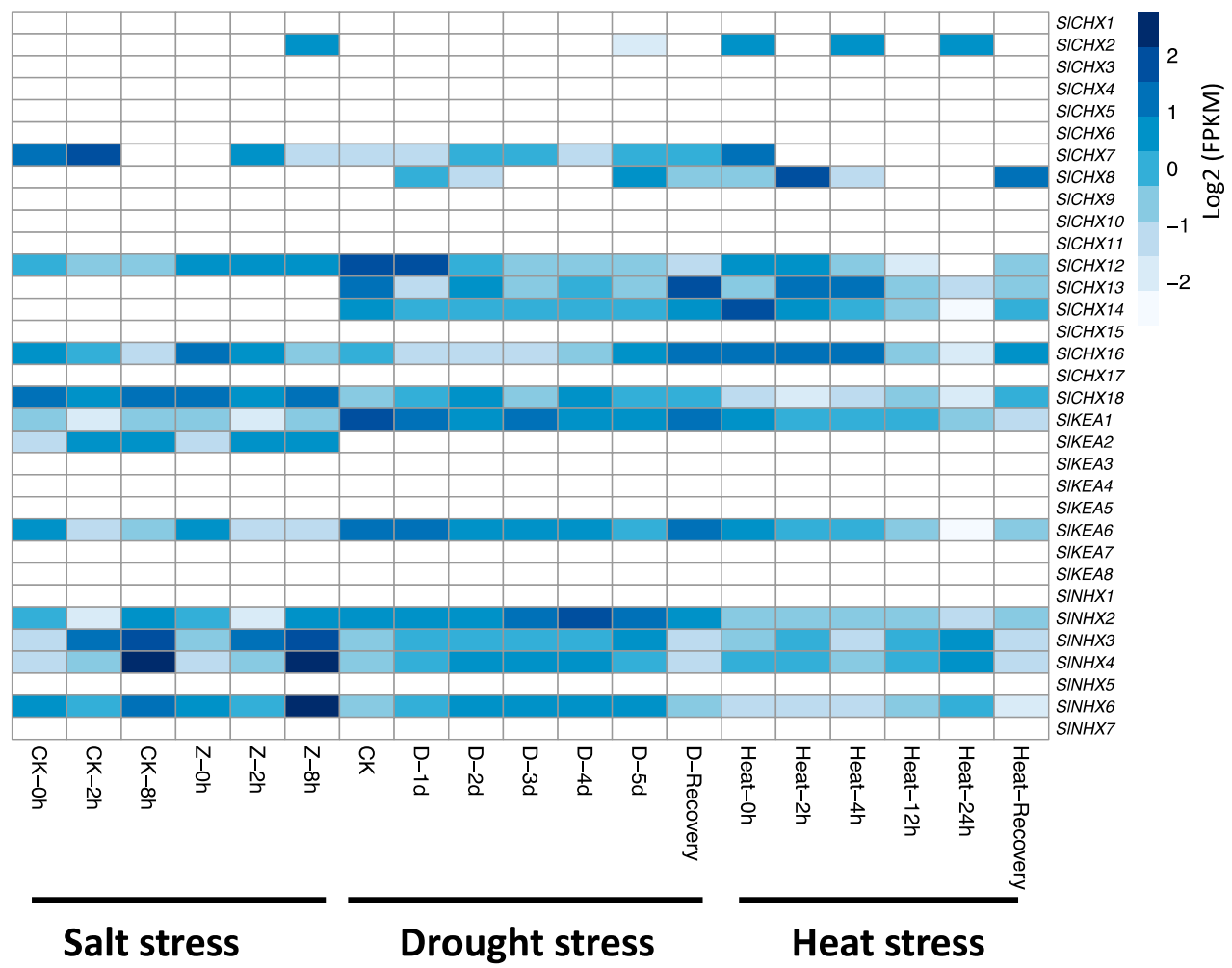

Figure 6. Gene expression analysis of SICPA genes in different tissues and various fruit developmental stages (A) and under heat, drought, and salinity conditions (B) from RNA-sequencing. The expression values were calculated by Log2 (FPKM) and presented according to the color code. White boxes represent no expression. 


\subsection{Expression Analysis of SICPA Genes under Heat, Drought, and Salinity Stresses}

The differential expression patterns of putative SICPA genes were further investigated under different abiotic stress conditions using the publicly available RNA-seq data (Figure 6B). Among the 33 SlCPAs, only 15 genes showed differential gene expression in response to salt, drought, and heat stress, suggesting their important role in abiotic stress tolerance. However, 16 genes were not expressed in response to salt, drought, and heat stress. Among the 15 differentially expressed genes, we also noted the specific regulation of SlCPAs genes in response to particular stress. For example, SlKEA2 was expressed only in response to salt stress, while SlCHX8, SlCHX813, and SlCHX14 were expressed in response to drought and heat stress but not in salt stress. Together, these results indicate their potential role in abiotic stress tolerance.

\subsection{Quantitative Expression Analysis of SICPAs under Salinity Stress}

The RNA-seq data were further validated by qRT-PCR analysis by exposing tomato plants to salt stress (Figure 7). Gene expression was analyzed at 0, 3, 6, 12, 24, 48, and $96 \mathrm{~h}$ time intervals in response to salt stress. Ten SlCPA genes (SlCHX14, SlCHX16, SlCHX18, SlKEA1, SlKEA2, SlKEA6, SlNHX2, SlNHX3, SlNHX4, and SlNHX6) were selected for qRT-PCR as they were highly upregulated in response to salt, drought, and heat stress (Figure 6B). In general, similar to RNA-seq, all the selected SICPA genes were highly upregulated (five to seven-fold) in response to salt stress at different time intervals. On average, the expression was significantly increased over time, with a maximum after $24 \mathrm{~h}$ of salt stress, but then decreased at $96 \mathrm{~h}$. However, the response of some genes was stronger than others, even after $3 \mathrm{~h}$ of salt stress. For example, SlCHX18, SlKEA6, and SlNHX3 were almost at the maximum level of their gene expression only after $3 \mathrm{~h}$ of salt stress.

\subsection{Co-Localization of SICPA Genes with QTLs (Quantitative Trait Loci) of Salt Stress-Related Phenotypes}

To gain more insight into the role of SlCPA genes in salt tolerance, SICPA genes were mapped with the previously reported salt-tolerance-related QTLs (Figure 8). These QTLs were reported previously based on the morpho-biochemical traits under salt stress, i.e., time to flower (Flw), time to ripe (RIP), leaf length (Leaf), leaf area (LA), dry shoot weight (DSW), number of fruits ripen (NFR), fruit weight (FW), fruit firmness (Firm), soluble solid content (SSC), $\mathrm{Na}^{+}$concentration in leaves (LNC), $\mathrm{K}^{+}$concentration in leaves (LKC), $\mathrm{Na}+$ concentration in the shoot $(\mathrm{SNC}), \mathrm{K}^{+}$concentration in the shoot $(\mathrm{SKC})$, the ratio of $\mathrm{K}^{+} / \mathrm{Na}^{+}$in leaves (LKN), $\mathrm{Cl}^{-}$accumulation in leaves, total $\mathrm{Na}^{+}$content (TN), and salt tolerance (ST). The gene names and physical position with respective co-localized QTL and linked marker name and physical position are provided in Table S5.

The co-localization results showed that chromosome one has thirteen salt-related QTLs. Among the thirteen salt-related QTLs on chromosome one, only four were co-localized with SlNHX1, SlKEA1, and SlNHX4. Both genes on chromosome two (i.e., SlCHX1 and SlCHX2) were co-localized with four QTLs (FW 2.1, NFr2.1, FLW2.1, and SSC2.1). Chromosome five had three SlCPA genes but only SlCHX5 was co-localized with the lkc5.1 and skc5.2 QTLs. Out of five SICPA genes on chromosome six, four SlCPAs genes were co-localized with the previously reported QTLs $[35,36]$. Chromosome seven has only one gene (SlKEA5), which showed co-localization with the SSC3.1. Chromosome eight has seven SICPA genes, but only two genes (SlKEA6 and SlCHX13) were co-localized with two QTLs. Chromosome nine has only one SICPA gene (SlCHX16), which co-localized with two QTLs. Chromosome 10 also has one SICPA gene (SlNHX7), which co-localized with three QTLs. Two genes on chromosome 11 (SlKEA8 and SlKEA7) coincided with the three QTLs (Firm11.2, SSC11.1, and FW11.1). Chromosome 12 has two SlCPA genes (SlCHX17 and SlCHX18) but only SlCHX18 was co-localized with the NFr12 (number of fruits ripen). The co-localization of SICPA genes with salt-related QTLs suggested a comprehensive role of the SICPA genes in different morpho-biochemical traits under salt stress. 

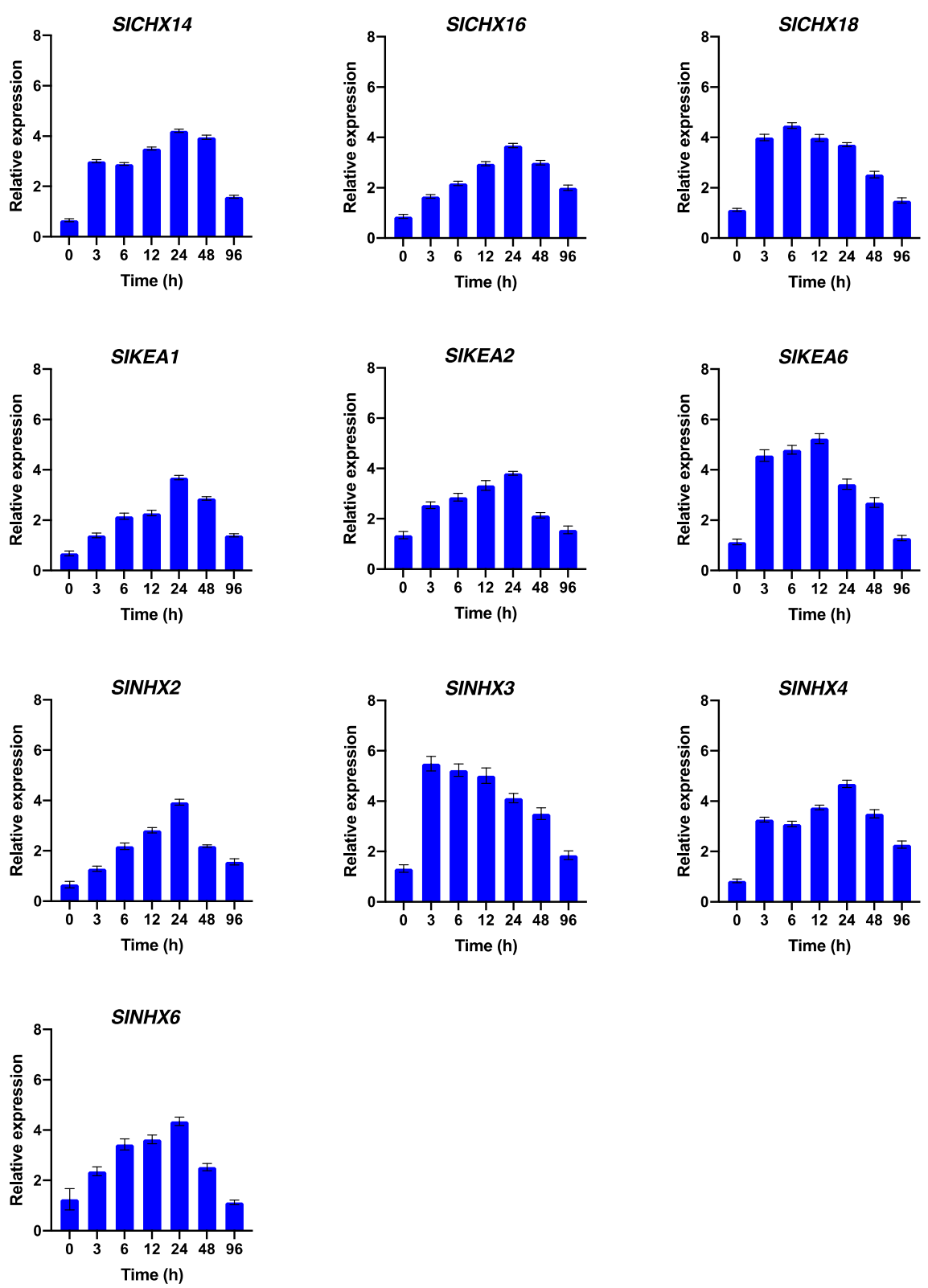

Figure 7. Expression profiles (qRT-PCR) of the SICPA genes at different time intervals $(0,3,6,12,24$, 48 , and $96 \mathrm{~h}$ ) under $250 \mathrm{mM} \mathrm{NaCl}$ treatments. The relative expressions at different stress treatment times were compared with the control $(0 \mathrm{~h})$.

\subsection{Protein-Protein Interaction and Gene Ontology Analysis}

The protein-protein interactions among the SlCPAs and the associated proteins were predicted by string database and modified in Cytoscape. The majority of the SlCPAs revealed strong protein-protein interaction networks, as depicted in Figure 9. Overall, the $\mathrm{CHX}$ subgroup members of SlCPAs possessed more protein-protein interactions than other subgroups. The members of the CPA protein family interact with other proteins, such as plasma membrane $\mathrm{H}^{+}$ATPase (LHA1), calcineurin B-like protein 1 (CBL1), CBL-interacting protein kinases (CIPK), salt overly sensitive (SOS1), PIN-Formed 2 (PIN2), HIGH-AFFINITY $\mathrm{K}^{+}$TRANSPORTER 1 (HKT1), and HIGH-AFFINITY $\mathrm{K}^{+}$TRANSPORTER 2 (HKT2). All these proteins are involved in salinity tolerance and support chloroplast against the reactive oxygen species and other pathways leading to abiotic stress tolerance. Our PPI networks provide essential evidence for understanding the functions of proteins with unknown functions. 

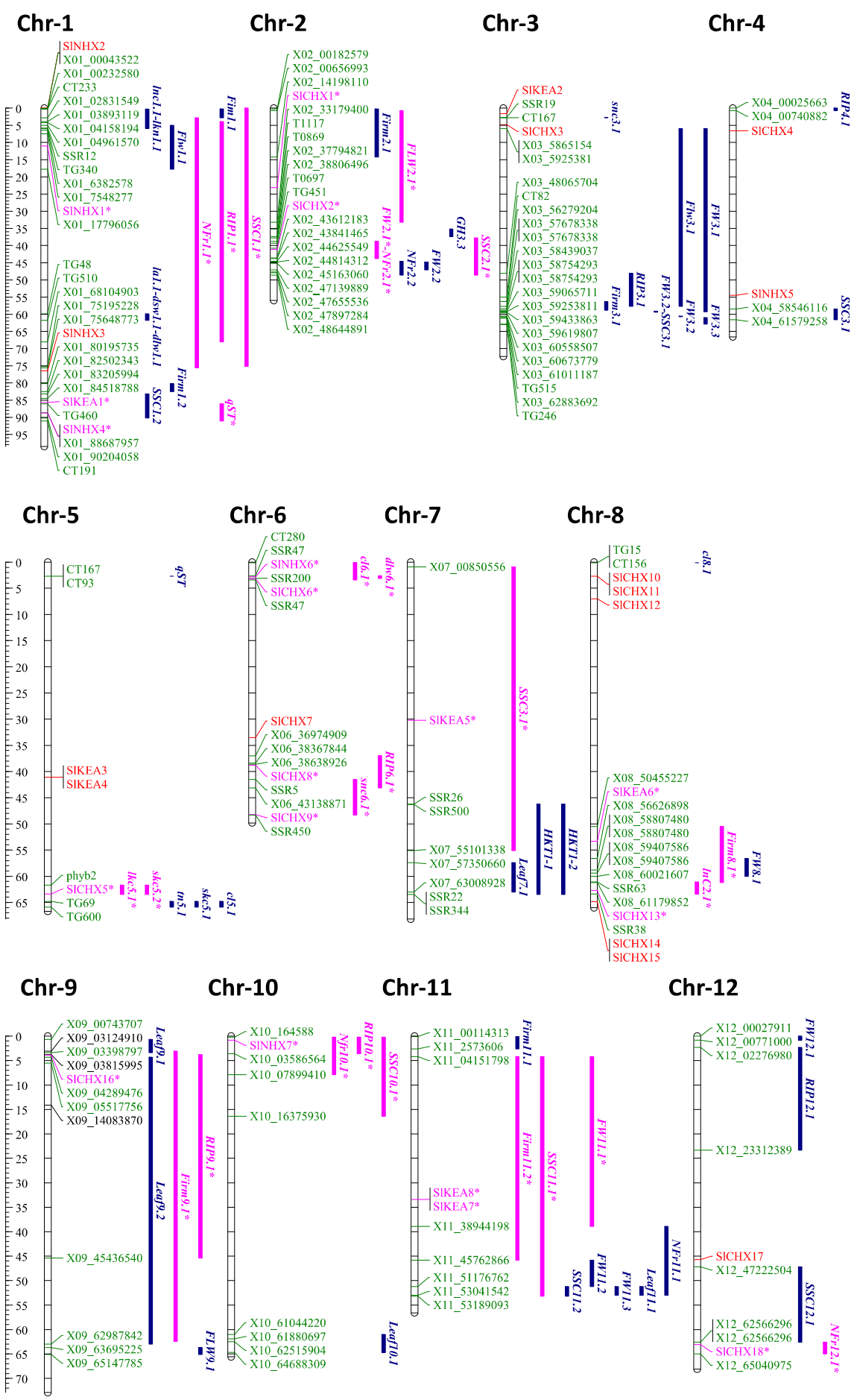

Figure 8. Co-localization of SlCPA genes with quantitative trait loci (QTLs) of salt stress-related phenotypes on the chromosomes of tomato. The scale represents the physical position of genes and QTL-linked markers in megabases $(\mathrm{Mb})$. These QTLs were reported previously based on the morpho-biochemical traits under salt stress; i.e., time to flower (Flw), time to ripe (RIP), leaf length (Leaf), leaf area (LA), dry shoot weight (DSW), number of fruits ripen (NFR), fruit weight (FW), fruit firmness (Firm), soluble solid content (SSC), $\mathrm{Na}^{+}$concentration in leaves (LNC), $\mathrm{K}^{+}$concentration in leaves (LKC), $\mathrm{Na}^{+}$concentration in the shoot (SNC), $\mathrm{K}^{+}$concentration in shoot (SKC), ratio of $\mathrm{K}^{+} / \mathrm{Na}^{+}$in leaves (LKN), $\mathrm{Cl}^{-}$accumulation in leaves, total $\mathrm{Na}^{+}$content (TN), and salt tolerance (ST). SICPA genes, which are not co-localized with any reported QTL, are highlighted with red color and QTLs in blue color, while SICPA genes and QTLs are in pink color with asterisks indicating that these SICPA genes co-localized with QTLs of salt stress-related phenotypes. 


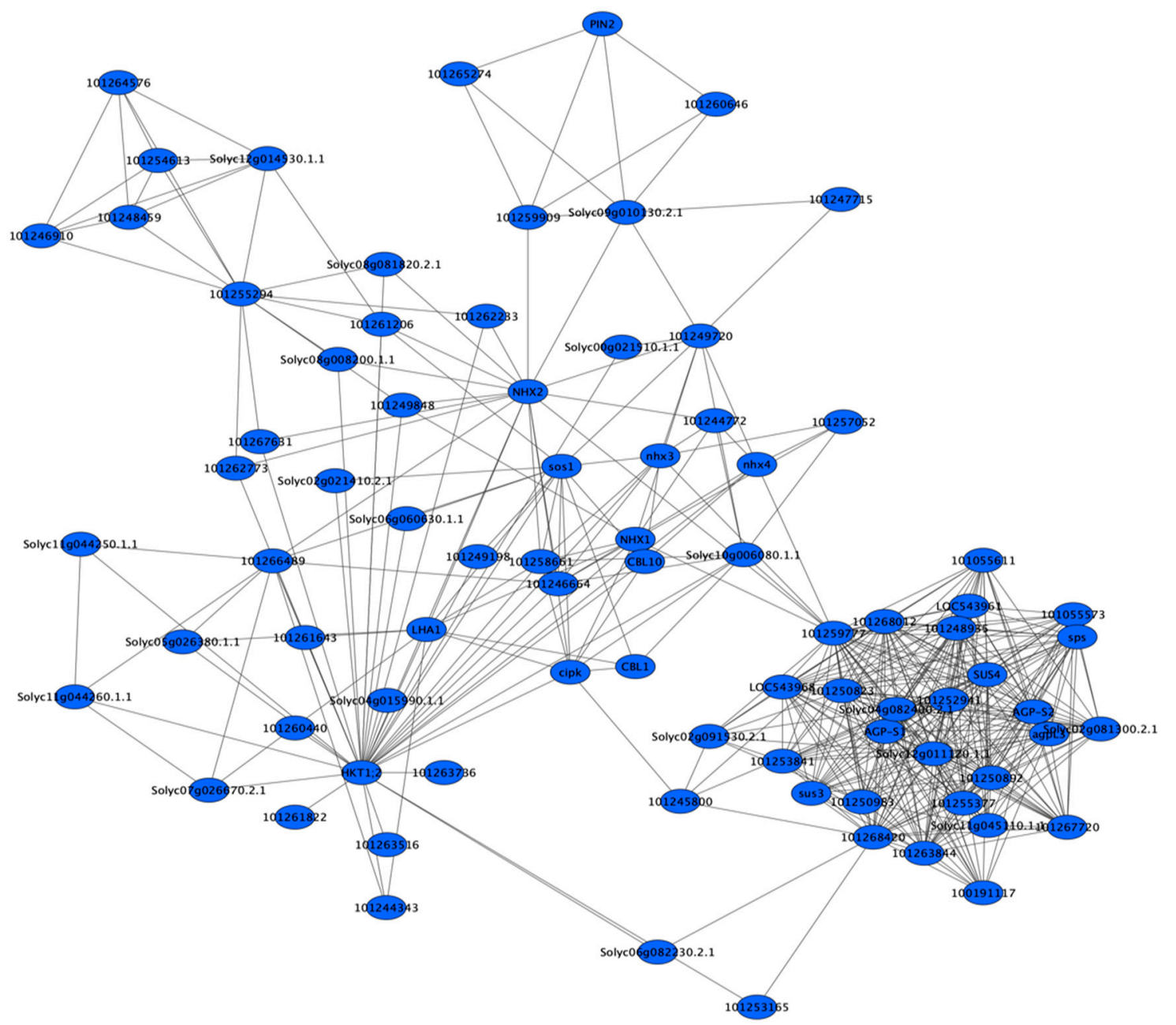

Figure 9. Network interactions of SlCPA proteins. Strong interaction network among SlCPA proteins and other related proteins supports their role in salinity tolerance.

Furthermore, the gene ontology (GO) terms analysis of CPAs in tomato revealed that they are involved in different biological and chemical processes (Figure 10). These functions are more prominently related to transport $(34 \%)$, homeostatic process $(18 \%)$, protein targeting $(15 \%)$, vacuolar transport $(15 \%)$, response to stress $(1.75 \%)$, transmembrane transport $(2.03 \%)$, vesicle-mediated transport $(2.03 \%)$, and a few others.

A

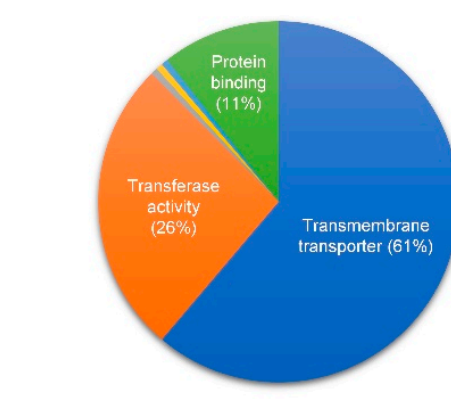

- Transmembrane transporter $=$ Peptidase activity - Ion binding
B

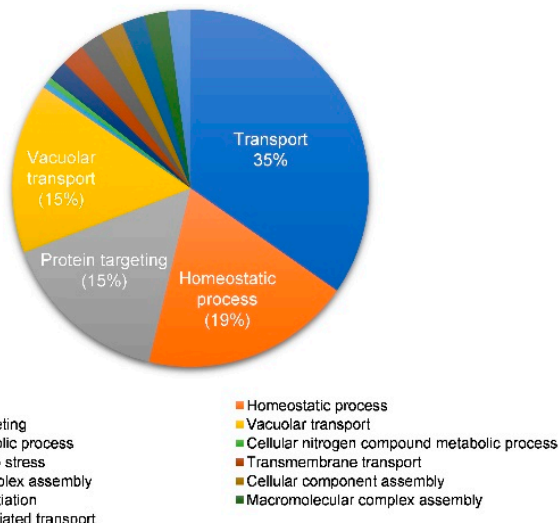

Figure 10. Gene ontology (GO) terms of SlCPA genes in tomato. Molecular function (A) and biological process (B) are shown in pie charts. Different GO terms are represented by different colors (\%). 


\section{Discussion}

In plants, ion concentration and $\mathrm{pH}$ balance feat are accomplished with a diverse array of transporters, especially the CPA proteins [1] that are localized in the plasma membrane as well as organelle membranes such as endosomes, vacuoles, and chloroplasts [3,7,8]. In this study, we revealed that tomato has 33 CPA members that could be classified into three main subgroups: CHX (18 members), NHX (7 members), and KEA (8 members). The CPA genes in tomato were lower in number as compared with the previously reported CPA genes in various species such as Triticum aestivum (107 genes), Raphanus sativus (60 genes), Brassica rapa (64 genes), Zea mays (33 genes), Arabidopsis thaliana (42 genes), Pyrus communis (53 genes), Oryza sativa (28 genes), Sorghum bicolor (28 genes), and Zea mays (33 genes) [4]. This might be attributed to the small and compact diploid genome of Solanum lycopersicum. Multiple studies investigating other gene families in tomato also revealed that tomato has fewer genes as compared with other plants [41-43]. However, similar to other plant species, the CHX subgroup of SIPCAs has the highest number of genes in tomato compared with KEA and NHX subgroups [4,5]. Furthermore, sub-classification of NHX, KEA, and CHX subgroups into different sub-clusters, such as N1-N2, K1-K2, and C1-C3, has also been observed in other plant species [4]. These observations suggest that CPAs are evolutionary conserved in plants.

The plant CPA1 (NHX) transporters are predicted to have 10-12 transmembrane domains, whereas the CPA2 (CHX and KEA) transporters are predicted to have 8-14 membrane spanning domains with a Pfam00999 domain for the $\mathrm{Na}^{+}, \mathrm{K}^{+} / \mathrm{H}^{+}$exchanger [3]. Studies have shown that CPA family members contain a catalytic conserved $\mathrm{Na}+/ \mathrm{H}+$ exchange domain in the $\mathrm{N}$ terminus [44-46] and our analysis of domains revealed that all SlCPA proteins contain the highly conserved N-terminal catalytic NHX domain, suggesting that tomato CPAs may have similar functions as described in other plant species.

CPA genes have been shown to regulate cellular $\mathrm{pH}$ and ion homeostasis and are involved in a wide range of physiological events, from vesicle trafficking to development $[3,46]$. However, some members of the CPAs comprise plasma membrane, vacuolar, and endosomal forms, and they have been identified to play an important role in salinity tolerance [47]. The role of numerous CPA genes in Arabidopsis and other plant species in salt tolerance has been established in earlier studies $[4,46,48]$. For example, NHX subgroup members of CPA genes have been shown to play a role in abiotic stress tolerance, including salinity stress, in different plant species [3,49-51]. Similarly, members of KEAs subgroup of Arabidopsis CPAs (AtKEA1, AtKEA2, and AtKEA5) and soybean CPA (GmKEAs) were upregulated during $\mathrm{Na}^{+}$and $\mathrm{K}^{+}$stresses [52,53]. Furthermore, the $\mathrm{CHX}$ subgroup of $C P A$ genes of Arabidopsis, including AtCHX13, AtCHX17, AtCHX21, and AtCHX23, were also reported to play a role in the salinity stress [53-55]. Our results highlighted that members of NHX, CHX, and KEA subgroups, but not all, may play an important role in countering the negative effect of salinity stress in the plant cell (Figures 5 and 6B). Co-localization of SICPAs genes with QTLs of salt stress-related phenotypes further support their role in salinity tolerance. Furthermore, in agreement with this observation, our qRT-PCR confirmed that SlCHX14, SlCHX16, SlCHX18, SlKEA1, SlKEA2, SlKEA6, SlNHX2, SlNHX3, SlNHX4, and SINHX6 were several folds up-regulated after salinity stress, supporting their potential role in salt tolerance in tomato. Interestingly, these genes also showed up-regulation in response to heat and drought (Figure 6B). However, similar to the grapevine, wheat, radish, maize, and soybean [12], not all SICPAs responded to salinity stress and other abiotic stresses. Furthermore, SICPA genes responded differentially depending on the intensity and type of stress treatment, suggesting that $S l C P A s$ may work together in response to particular stimuli, and may participate in long-term resistance to abiotic stresses. However, further molecular and biochemical studies are required to validate SlCPAs function in multiple abiotic stresses and understand the underlying molecular mechanism.

Tandem duplication, polyploidy, and segmental duplications primarily contribute to creating new gene families (e.g., MYB, WRKY, and CytP450 gene families) in the evolution of genome and genetic systems [56]. Five segmental duplications were found in the 
tomato genome (SlNHX4/SlNHX7, SlKEA1/SlKEA7, SlKEA2/SlKEA3, and SlCHX2/SlCHX11, SlCHX5/SlCHX17) (Figure 3). Gene duplications occurred in these genes because the identities of the genes flanking both sides of the paralogous tomato CPA genes were found to be absolutely conserved and located on duplicated segments on two different chromosomes. Segmental duplication was also predicted as the main driving force for the amplification of the SITGL gene family in tomato. SlCPAs carry TATA and CAAT box core cis-elements, light-responsive, environmental stress-related, development-responsive, and phytohormones response elements in their promoters (Figure 5), suggesting that SlCPAs are likely to play important roles in response to developmental and environmental cues. Interestingly, these duplicated genes carry different cis-elements in their promoters, suggesting their functional divergence in response to particular stimuli. However, among the duplicated genes, only one gene responded to abiotic stress (Figure 6B). For example, SIKEA1 responded to all abiotic stresses but not SIKEA7; SINHX4 responded to all abiotic stresses but not $S I N H X 7$, further supporting the functional divergence of duplicated genes.

The network interactions of SlCPA proteins revealed that SlCPAs were predicted to interact with many other proteins such as SOS1, LHA1, CIPK, CBL1, PIN2, HKT1, and HKT2 (Figure 9). SOS1 is the pivotal kinase of the SOS pathway involved in the regulation of ion transport under abiotic stress, especially the salt stress [55]. Similarly, LHA1 is also a member of the plasma membrane $\mathrm{H}^{+}$ATPase that plays a critical role in plant adaptation to saline conditions, as it generates proton gradient that actively transports nutrients by $\mathrm{H}^{+}$-symport [57]. It has been previously observed that HKT1 and HKT2 proteins are expressed during high salinity conditions to neutralize the excess $\mathrm{Na}^{+}$ion in xylem tissues [58]. Recent studies have found that CBLs interact with CIPKs to form a CBL-CIPK signaling network that takes part in the transport of ions and participates in multiple abiotic stresses in plants, including drought, heat, cold and salinity [59-61]. These results suggest that SlCPA may belong to larger protein complexes, thus regulating abiotic stress tolerance with their partners. However, further comprehensive studies are required to confirm these interactions.

In brief, our study highlighted the implication of tomato CPAs in abiotic stress adaptation. This study strongly recommends the comprehensive dissection of the biological and cellular function of tomato CPAs, which will eventually lead to a long-term improvement of abiotic stress tolerance in tomato.

\section{Conclusions}

In this study, a total of 33 members of the CPA gene family, comprising NHX, KEA, and $C H X$ subgroups, were identified in the diploid genome of tomato. Tomato CPAs are unevenly distributed on their chromosomes, and segmental duplication contributed to the evolution of the CPAs family. Cis-elements analysis discovered several plant developments, stress related, hormonal, and light response cis-elements in the promoter of tomato CPAs, but each member had peculiar types and numbers. Furthermore, gene expression analysis exhibited that 15 members of the tomato $C P A$ family are differentially regulated in response to abiotic stresses. Several tomato CPA genes were co-localized with QTLs of salt stressrelated phenotypes, which disclosed that tomato CPAs play roles in abiotic stress tolerance. Tomato CPAs were predicted to interact with proteins, such as SOS1, LHA1, CIPK, CBL1, PIN2, HKT1, and HKT2, that have been previously described as important players in response to salinity stress. Thus, our study helps to lay the foundation for the functional characterization of the tomato CPA gene family by overexpression and knockdown/out using RNAi or CRISPR-Cas9 genome editing. This study also provides a fundamental understanding of tomato $C P A s$ in conferring abiotic stress resistance in tomato and will be useful for the long-term improvement of stress tolerance in tomato.

Supplementary Materials: The following are available online at https:/ / www.mdpi.com/article/ 10.3390/agronomy12020245/s1: Figure S1: Discovered motif logos in SlCPA proteins; Table S1: Physiochemical properties of SlCPA genes; Table S2: List of Cis-elements in the promoter of SlCPA genes; and Table S3: List of primers used in the study; Table S4: Analysis and annotation of SlCPA 
proteins motifs of Figure 2A; Table S5: Description of co-localized genes with QTLs and linked marker position.

Author Contributions: Conceptualization, Z.H., H.K., methodology, Z.H., S.S. and S.H.S.; software, M.K.N., M.I., Z.H., M.R. and H.K.; validation, Z.H., S.H.S. and A.I.; formal analysis, Z.H., E.W., M.A.M.; investigation, Z.H., M.I., M.K.N.; resources, H.K., S.S.A., S.A., M.R.; data curation, S.S.A., M.R., M.A.M. and S.A.; writing-original draft preparation, Z.H. and S.S.; writing-review and editing, E.W., S.S. and Z.H.; visualization, Z.H., H.K., S.S., S.S.A.; supervision, Z.H. and S.S.; project administration, Z.H. and S.S.; funding acquisition, S.S. and E.W. All authors have read and agreed to the published version of the manuscript.

Funding: This research received no external funding.

Institutional Review Board Statement: Not applicable.

Informed Consent Statement: Not applicable.

Data Availability Statement: The authors confirm that the data supporting the findings of this study are available within the article and its Supplementary Materials.

Acknowledgments: The authors are grateful to the Centre for Biotechnology and Microbiology, University of Swat, Pakistan, for technical support.

Conflicts of Interest: The authors declare no conflict of interest. The funders had no role in the design of the study; in the collection, analyses, or interpretation of data; in the writing of the manuscript, or in the decision to publish the results.

\section{References}

1. Brett, C.L.; Donowitz, M.; Rao, R. Evolutionary origins of eukaryotic sodium/proton exchangers. Am. J. Physiol. Cell Physiol. 2005, 288, C223-C239. [CrossRef] [PubMed]

2. Saier, M.H., Jr. A functional-phylogenetic classification system for transmembrane solute transporters. Microbiol. Mol. Biol. Rev. 2000, 64, 354-411. [CrossRef]

3. Chanroj, S.; Wang, G.; Venema, K.; Zhang, M.W.; Delwiche, C.F.; Sze, H. Conserved and diversified gene families of monovalent cation $/ \mathrm{h}(+)$ antiporters from algae to flowering plants. Front. Plant Sci. 2012, 3, 25. [CrossRef] [PubMed]

4. Ye, C.Y.; Yang, X.; Xia, X.; Yin, W. Comparative analysis of cation/proton antiporter superfamily in plants. Gene 2013, 521, 245-251. [CrossRef] [PubMed]

5. Sharma, H.; Taneja, M.; Upadhyay, S.K. Identification, characterization and expression profiling of cation-proton antiporter superfamily in Triticum aestivum L. and functional analysis of TaNHX4-B. Genomics 2020, 112, 356-370. [CrossRef]

6. Masrati, G.; Dwivedi, M.; Rimon, A.; Gluck-Margolin, Y.; Kessel, A.; Ashkenazy, H.; Mayrose, I.; Padan, E.; Ben-Tal, N. Broad phylogenetic analysis of cation/proton antiporters reveals transport determinants. Nat. Commun. 2018, 9, 4205. [CrossRef] [PubMed]

7. An, R.; Chen, Q.J.; Chai, M.F.; Lu, P.L.; Su, Z.; Qin, Z.X.; Chen, J.; Wang, X.C. AtNHX8, a member of the monovalent cation: Proton antiporter-1 family in Arabidopsis thaliana, encodes a putative Li/H antiporter. Plant J. 2007, 49, 718-728. [CrossRef] [PubMed]

8. Very, A.A.; Sentenac, H. Molecular mechanisms and regulation of $\mathrm{K}^{+}$transport in higher plants. Annu. Rev. Plant Biol. 2003, 54, 575-603. [CrossRef] [PubMed]

9. Mäser, P.; Thomine, S.; Schroeder, J.I.; Ward, J.M.; Hirschi, K.; Sze, H.; Talke, I.N.; Amtmann, A.; Maathuis, F.J.; Sanders, D.; et al. Phylogenetic relationships within cation transporter families of Arabidopsis. Plant Physiol. 2001, 126, 1646-1667. [CrossRef]

10. Wang, Y.; Ying, J.; Zhang, Y.; Xu, L.; Zhang, W.; Ni, M.; Zhu, Y.; Liu, L. Genome-Wide Identification and Functional Characterization of the Cation Proton Antiporter (CPA) Family Related to Salt Stress Response in Radish (Raphanus sativus L.). Int. J. Mol. Sci. 2020, 21, 8262. [CrossRef] [PubMed]

11. Zeng, Y.; Li, Q.; Wang, H.; Zhang, J.; Du, J.; Feng, H.; Blumwald, E.; Yu, L.; Xu, G. Two NHX-type transporters from Helianthus tuberosus improve the tolerance of rice to salinity and nutrient deficiency stress. Plant Biotechnol. J. 2018, 16, 310-321. [CrossRef]

12. Ma, Y.; Wang, J.; Zhong, Y.; Cramer, G.; Cheng, M. Genome-wide analysis of the cation/proton antiporter (CPA) super family genes in grapevine (Vitis vinifera L.). Plant OMICS 2015, 8, 300-311.

13. Zhou, H.; Qi, K.; Liu, X.; Yin, H.; Wang, P.; Chen, J.; Wu, J.; Zhang, S. Genome-wide identification and comparative analysis of the cation proton antiporters family in pear and four other Rosaceae species. Mol. Genet. Genom. 2016, 291, 1727-1742. [CrossRef] [PubMed]

14. Arumuganathan, K.E.E.; Earle, E. Nuclear DNA Content of Some Important Plant Species. Plant Mol. Biol. Report. 1991, 9, 208-218. [CrossRef]

15. Tomato Genome, C. The tomato genome sequence provides insights into fleshy fruit evolution. Nature 2012, 485, 635-641. [CrossRef] [PubMed] 
16. Bai, Y.; Kissoudis, C.; Yan, Z.; Visser, R.G.F.; van der Linden, G. Plant behaviour under combined stress: Tomato responses to combined salinity and pathogen stress. Plant J. 2018, 93, 781-793. [CrossRef]

17. Pettersen, E.F.; Goddard, T.D.; Huang, C.C.; Couch, G.S.; Greenblatt, D.M.; Meng, E.C.; Ferrin, T.E. UCSF Chimera-a visualization system for exploratory research and analysis. J. Comput. Chem. 2004, 25, 1605-1612. [CrossRef] [PubMed]

18. Eddy, S.R. Accelerated Profile HMM Searches. PLoS Comput. Biol. 2011, 7, e1002195. [CrossRef]

19. Finn, R.D.; Clements, J.; Eddy, S.R. HMMER web server: Interactive sequence similarity searching. Nucleic Acids Res. 2011, 39, W29-W37. [CrossRef] [PubMed]

20. Zdobnov, E.M.; Apweiler, R. InterProScan-an integration platform for the signature-recognition methods in InterPro. Bioinformation 2001, 17, 847-848. [CrossRef] [PubMed]

21. Gasteiger, E.; Gattiker, A.; Hoogland, C.; Ivanyi, I.; Appel, R.D.; Bairoch, A. ExPASy: The proteomics server for in-depth protein knowledge and analysis. Nucleic Acids Res. 2003, 31, 3784-3788. [CrossRef] [PubMed]

22. Yu, C.S.; Cheng, C.W.; Su, W.C.; Chang, K.C.; Huang, S.W.; Hwang, J.K.; Lu, C.H. CELLO2GO: A web server for protein subCELlular LOcalization prediction with functional gene ontology annotation. PLoS ONE 2014, 9, e99368.

23. Chen, Y.; Yu, P.; Luo, J.; Jiang, Y. Secreted protein prediction system combining CJ-SPHMM, TMHMM, and PSORT. Mamm. Genome 2003, 14, 859-865. [CrossRef]

24. Machanick, P.; Bailey, T.L. MEME-ChIP: Motif analysis of large DNA datasets. Bioinformatics 2011, 27, 1696-1697. [CrossRef] [PubMed]

25. Tamura, K.; Stecher, G.; Peterson, D.; Filipski, A.; Kumar, S. MEGA6: Molecular Evolutionary Genetics Analysis version 6.0. Mol. Biol. Evol. 2013, 30, 2725-2729. [CrossRef] [PubMed]

26. Letunic, I.; Bork, P. Interactive Tree of Life (iTOL) v4: Recent updates and new developments. Nucleic Acids Res. 2019, 47, W256-W259. [CrossRef]

27. Wang, Y.; Tang, H.; Debarry, J.D.; Tan, X.; Li, J.; Wang, X.; Lee, T.H.; Jin, H.; Marler, B.; Guo, H.; et al. MCScanX: A toolkit for detection and evolutionary analysis of gene synteny and collinearity. Nucleic Acids Res. 2012, 40, e49. [CrossRef]

28. Chen, C.; Chen, H.; Zhang, Y.; Thomas, H.R.; Frank, M.H.; He, Y.; Xia, R. TBtools: An Integrative Toolkit Developed for Interactive Analyses of Big Biological Data. Mol. Plant 2020, 13, 1194-1202. [CrossRef] [PubMed]

29. Imran, M.; Shafiq, S.; Farooq, M.A.; Naeem, M.K.; Widemann, E.; Bakhsh, A.; Jensen, K.B.; Wang, R.R. Comparative Genome-wide Analysis and Expression Profiling of Histone Acetyltransferase (HAT) Gene Family in Response to Hormonal Applications, Metal and Abiotic Stresses in Cotton. Int. J. Mol. Sci. 2019, 20, 5311. [CrossRef] [PubMed]

30. Lescot, M.; Dehais, P.; Thijs, G.; Marchal, K.; Moreau, Y.; Van de Peer, Y.; Rouze, P.; Rombauts, S. PlantCARE, a database of plant cis-acting regulatory elements and a portal to tools for in silico analysis of promoter sequences. Nucleic Acids Res. 2002, 30, 325-327. [CrossRef]

31. Higo, K.; Ugawa, Y.; Iwamoto, M.; Korenaga, T. Plant cis-acting regulatory DNA elements (PLACE) database: 1999. Nucleic Acids Res. 1999, 27, 297-300. [CrossRef]

32. Hoagland, D.R.; Arnon, D.I. The Water-Culture Method for Growing Plants without Soil. Circ. Calif. Agric. Exp. Stn. 1950, 347,32

33. Shafiq, S.; Ali, A.; Sajjad, Y.; Zeb, Q.; Shahzad, M.; Khan, A.R.; Nazir, R.; Widemann, E. The Interplay between Toxic and Essential Metals for Their Uptake and Translocation Is Likely Governed by DNA Methylation and Histone Deacetylation in Maize. Int. J. Mol. Sci. 2020, 21, 6959. [CrossRef]

34. Szklarczyk, D.; Franceschini, A.; Kuhn, M.; Simonovic, M.; Roth, A.; Minguez, P.; Doerks, T.; Stark, M.; Muller, J.; Bork, P.; et al. The STRING database in 2011: Functional interaction networks of proteins, globally integrated and scored. Nucleic Acids Res. 2011, 39, D561-D568. [CrossRef] [PubMed]

35. Villalta, I.; Reina-Sánchez, A.; Bolarín, M.C.; Cuartero, J.; Belver, A.; Venema, K.; Carbonell, E.; Asins, M. Genetic analysis of Na+ and $\mathrm{K}+$ concentrations in leaf and stem as physiological components of salt tolerance in Tomato. TAG. Theor. Appl. Genet. Theor. Und Angew. Genet. 2008, 116, 869-880. [CrossRef] [PubMed]

36. Diouf, I.A.; Derivot, L.; Bitton, F.; Pascual, L.; Causse, M. Water Deficit and Salinity Stress Reveal Many Specific QTL for Plant Growth and Fruit Quality Traits in Tomato. Front. Plant Sci. 2018, 9, 279. [CrossRef] [PubMed]

37. Foolad, M.R.; Zhang, L.P.; Lin, G.Y. Identification and validation of QTLs for salt tolerance during vegetative growth in tomato by selective genotyping. Genome 2001, 44, 444-454. [CrossRef]

38. Asins, M.J.; Villalta, I.; Aly, M.M.; Olías, R.; Morales, P.A.D.E.; Huertas, R.; Li, J.; Jaime-Pérez, N.; Haro, R.; Raga, V.; et al. Two closely linked tomato HKT coding genes are positional candidates for the major tomato QTL involved in $\mathrm{Na}^{+} / \mathrm{K}^{+}$homeostasis. Plant Cell Environ. 2013, 36, 1171-1191. [CrossRef]

39. Imran, M.; Shafiq, S.; Naeem, M.K.; Widemann, E.; Munir, M.Z.; Jensen, K.B.; Wang, R.R. Histone Deacetylase (HDAC) Gene Family in Allotetraploid Cotton and Its Diploid Progenitors: In Silico Identification, Molecular Characterization, and Gene Expression Analysis under Multiple Abiotic Stresses, DNA Damage and Phytohormone Treatments. Int. J. Mol. Sci. 2020, $21,321$. [CrossRef]

40. Voorrips, R.E. MapChart: Software for the graphical presentation of linkage maps and QTLs. J. Hered. 2002, 93, 77-78. [CrossRef] [PubMed]

41. Islam, S.; Rahman, I.A.; Islam, T.; Ghosh, A.A.-O. Genome-wide identification and expression analysis of glutathione S-transferase gene family in tomato: Gaining an insight to their physiological and stress-specific roles. PLoS ONE 2017, 12, e0187504. [CrossRef] 
42. Jin, J.F.; Wang, Z.Q.; He, Q.Y.; Wang, J.Y.; Li, P.F.; Xu, J.M.; Zheng, S.J.; Fan, W.; Yang, J.L. Genome-wide identification and expression analysis of the NAC transcription factor family in tomato (Solanum lycopersicum) during aluminum stress. BMC Genom. 2020, 21, 288. [CrossRef]

43. Fan, Y.; Yang, W.; Yan, Q.; Chen, C.; Li, J. Genome-Wide Identification and Expression Analysis of the Protease Inhibitor Gene Families in Tomato. Genes 2019, 11, 1. [CrossRef]

44. Czerny, D.D.; Padmanaban, S.; Anishkin, A.; Venema, K.; Riaz, Z.; Sze, H. Protein architecture and core residues in unwound alpha-helices provide insights to the transport function of plant AtCHX17. Biochim. Biophys. Acta 2016, 1858, 1983-1998. [CrossRef] [PubMed]

45. Sze, H.; Padmanaban, S.; Cellier, F.; Honys, D.; Cheng, N.H.; Bock, K.W.; Conejero, G.; Li, X.; Twell, D.; Ward, J.M.; et al. Expression patterns of a novel AtCHX gene family highlight potential roles in osmotic adjustment and $\mathrm{K}+$ homeostasis in pollen development. Plant Physiol. 2004, 136, 2532-2547. [CrossRef]

46. Padmanaban, S.; Chanroj, S.; Kwak, J.M.; Li, X.; Ward, J.M.; Sze, H. Participation of endomembrane cation/H+ exchanger AtCHX20 in osmoregulation of guard cells. Plant Physiol. 2007, 144, 82-93. [CrossRef]

47. Dong, W.; Li, D.L.; Qiu, N.W.; Song, Y.G. The functions of plant cation/proton antiporters. Biol. Plant. 2018, 62, 421-427. [CrossRef]

48. Mottaleb, S.A.; Rodriguez-Navarro, A.; Haro, R. Knockouts of Physcomitrella patens CHX1 and CHX2 transporters reveal high complexity of potassium homeostasis. Plant Cell Physiol. 2013, 54, 1455-1468. [CrossRef] [PubMed]

49. Apse, M.P.; Aharon, G.S.; Snedden, W.A.; Blumwald, E. Salt tolerance conferred by overexpression of a vacuolar $\mathrm{Na}^{+} / \mathrm{H}^{+}$antiport in Arabidopsis. Science 1999, 285, 1256-1258. [CrossRef]

50. Apse, M.P.; Sottosanto, J.B.; Blumwald, E. Vacuolar cation/H+ exchange, ion homeostasis, and leaf development are altered in a T-DNA insertional mutant of AtNHX1, the Arabidopsis vacuolar $\mathrm{Na}^{+} / \mathrm{H}^{+}$antiporter. Plant J. 2003, 36, 229-239. [CrossRef] [PubMed]

51. Bassil, E.; Ohto, M.; Esumi, T.; Tajima, H.; Zhu, Z.; Cagnac, O.; Belmonte, M.; Peleg, Z.; Yamaguchi, T.; Blumwalda, E. The Arabidopsis intracellular $\mathrm{Na}^{+} / \mathrm{H}^{+}$antiporters NHX5 and NHX6 are endosome associated and necessary for plant growth and development. Plant Cell 2011, 23, 224-239. [CrossRef] [PubMed]

52. Chen, H.-T.; Chen, X.; Wu, B.-Y.; Yuan, X.-X.; Zhang, H.-M.; Cui, X.-Y.; Liu, X.-Q. Whole-genome identification and expression analysis of $\mathrm{K}+$ efflux antiporter (KEA) and $\mathrm{Na}^{+} / \mathrm{H}^{+}$antiporter (NHX) families under abiotic stress in soybean. J. Integr. Agric. 2015, 14, 1171-1183. [CrossRef]

53. Zhao, J.; Cheng, N.H.; Motes, C.M.; Blancaflor, E.B.; Moore, M.; Gonzales, N.; Padmanaban, S.; Sze, H.; Ward, J.M.; Hirschi, K.D. AtCHX13 is a plasma membrane $\mathrm{K}+$ transporter. Plant Physiol. 2008, 148, 796-807. [CrossRef] [PubMed]

54. Hall, D.; Evans, A.R.; Newbury, H.J.; Pritchard, J. Functional analysis of CHX21: A putative sodium transporter in Arabidopsis J. Exp. Bot. 2006, 57, 1201-1210. [CrossRef] [PubMed]

55. Qiu, Q.S.; Guo, Y.; Dietrich, M.A.; Schumaker, K.S.; Zhu, J.K. Regulation of SOS1, a plasma membrane Na+/H+ exchanger in Arabidopsis thaliana, by $\mathrm{SOS}_{2}$ and $\mathrm{SOS}_{3}$. Proc. Natl. Acad. Sci. USA 2002, 99, 8436-8441. [CrossRef] [PubMed]

56. Cannon, S.B.; Mitra, A.; Baumgarten, A.; Young, N.D.; May, G. The roles of segmental and tandem gene duplication in the evolution of large gene families in Arabidopsis thaliana. BMC Plant Biol. 2004, 4, 10. [CrossRef] [PubMed]

57. Xu, W.; Jia, L.; Shi, W.; Baluska, F.; Kronzucker, H.J.; Liang, J.; Zhang, J. The Tomato 14-3-3 protein TFT4 modulates H+ efflux, basipetal auxin transport, and the PKS5-J3 pathway in the root growth response to alkaline stress. Plant Physiol. 2013, 163, 1817-1828. [CrossRef] [PubMed]

58. Zhang, W.D.; Wang, P.; Bao, Z.; Ma, Q.; Duan, L.J.; Bao, A.K.; Zhang, J.L.; Wang, S.M. SOS1, HKT1;5, and NHX1 Synergistically Modulate $\mathrm{Na}^{+}$Homeostasis in the Halophytic Grass Puccinellia tenuiflora. Front. Plant Sci. 2017, 8, 576. [CrossRef] [PubMed]

59. Weinl, S.; Kudla, J. The CBL-CIPK Ca ${ }^{2+}$-decoding signaling network: Function and perspectives. New Phytol. 2009, 184, 517-528. [CrossRef]

60. Cheong, Y.H.; Kim, K.N.; Pandey, G.K.; Gupta, R.; Grant, J.J.; Luan, S. CBL1, a calcium sensor that differentially regulates salt, drought, and cold responses in Arabidopsis. Plant Cell 2003, 15, 1833-1845. [CrossRef] [PubMed]

61. Jamshaid Hussain, G.B.; Shafiq, S. Calcium Dependent Protein Kinases in Plants; Wiley-Blackwell: Hoboken, NJ, USA, 2021. 